\title{
Botulinum toxin type A treatment of cerebral palsy: an integrated approach
}

\author{
Guy Molenaers ${ }^{a}$, Kaat Desloovere ${ }^{b}$, Marijke Eyssen ${ }^{c}$, Jos Decat ${ }^{d}$, Ilse Jonkers ${ }^{d}$ and \\ Paul De Cock
}

${ }^{a}$ Department of Orthopaedic Surgery, ${ }^{b}$ Gait Analysis Laboratory, 'Department of Neuro Paediatrics, and "Department of Physical Therapy, Faculty of Physical Education and Physical Therapy, University Hospital Leuven, Belgium

\author{
Correspondence to Dr Guy Molenaers at Department of Orthopaedic Surgery, University \\ Hospital Leuven, UZ Pellenberg, Weligerveld 1, 3212 Pellenberg, Belgium \\ Tel: +321633 8800; fax: +3216338824
}

\begin{abstract}
We have applied a multilevel approach to the management of spasticity associated with cerebral palsy (CP). All of the following factors are important in forming an integrated strategy for botulinum toxin type A (BTX-A) therapy: the timing of injections, patient selection, multilevel BTX-A treatment, optimal dosage and injection technique, follow-up treatment and objective measurements of functional outcome. Data on all these factors are presented here. CP patients had a mean age of 6.5 years $(n=315)$, and the dose of BTX-A (BOTOX ${ }^{\oplus}$ ) ranged from 2 to $29 \mathrm{U} / \mathrm{kg}$ body weight $(n=156)$. The combination of muscles injected in our multilevel approach differed for patients with diplegia, hemiplegia and quadriplegia: patients with hemiplegia received injections in the gastrocnemius and medial hamstrings; this combination was extended to the adductors for patients with diplegia and quadriplegia $(n=156)$. For patients with quadriplegia, muscles in a three-level (gastrocnemius, medial hamstrings, adductors and iliopsoas) or two-level (excluding the gastrocnemius) combination were also frequently injected. The duration of effect of BTX-A treatment was mainly determined by follow-up treatment consisting of: serial casting, day and night orthoses and physiotherapy. No major side effects of BTX-A were reported. This integrated approach appears to prolong the duration of BTX-A treatment, resulting in a duration of about 1 year between injections. Eur J Neurol 6 (suppl 4):S51S57 (1) Lippincott Williams \& Wilkins
\end{abstract}

Keywords: cerebral palsy, botulinum toxin type A, spasticity, physiotherapy, serial casting, children

\section{INTRODUCTION}

The use of botulinum toxin type A (BTX-A) in the management of paediatric gait disorders is now widely accepted. In the University Hospital of Pellenberg, we have applied a multilevel approach to the management of spasticity associated with cerebral palsy (CP). This article outlines the importance of using an integrated approach for BTX-A injections in the treatment of children with CP. BTX-A injections cannot be viewed in isolation as they are only one aspect of an integrated approach towards the multidisciplinary treatment of children with $\mathrm{CP}$.

Over the past few years of using BTX-A to treat children with $\mathrm{CP}$ at the University Hospital of Pellenberg, the necessity for an integrated approach has become apparent. Indeed, this strategy for BTX-A treatment has been developed continuously over the past 4 years. The importance of the various aspects of this strategy are based on: the objective evaluation

1351-5101 두 1999 Blackwell Science Ltd of BTX-A treatment using full gait analysis; feedback from the physiotherapists treating the patients postBTX-A injection; and a multidisciplinary postinjection follow-up programme.

At present, the main factors that form the integrated strategy for BTX-A treatment are:

- the correct timing for BTX-A injections;

- an appropriate patient selection procedure, i.e. one based upon objective measurements;

- multilevel BTX-A treatment (if indicated by the objective measurements);

- an optimal dose and injection technique;

- optimal follow-up treatment (e.g. combined serial casting, day and night orthoses and physiotherapy);

- objective measurements of functional outcome.

Only when all these aspects are properly addressed for the treatment of a child with $\mathrm{CP}$, is there assurance

European Journal of Neurology 1999, Vol 6 (suppl 4)

S51 


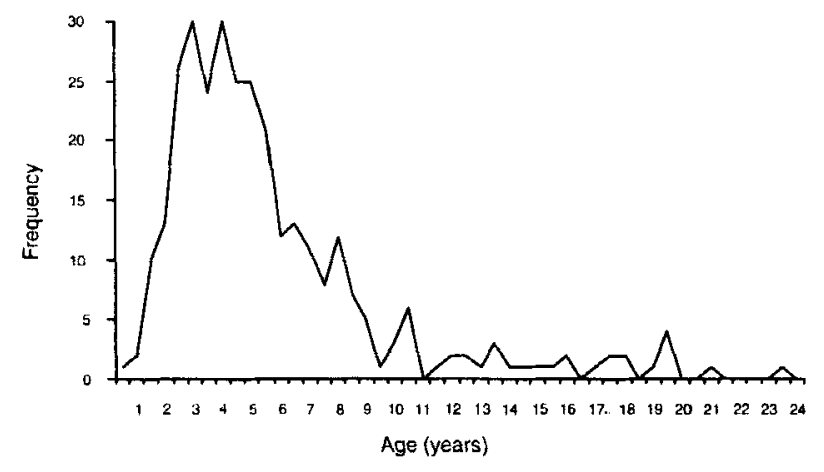

FIGURE 1. Frequency of age of 316 children with cerebral palsy at time of first injection with botulinum toxin type $A$ between February 1996 and April 1999

of a successful BTX-A treatment outcome. Until recently, it had not been possible to demonstrate the importance of each of these aspects using proper prospective studies, although each factor was thought to play an important role in the success of BTX-A treatment.

Each part of the integrated approach will be highlighted within this article and illustrated using data collected during 4 years' administering BTX-A therapy at the University Hospital of Pellenberg, together with preliminary results from an objective evaluation of BTX-A treatment.

\section{TIMING OF BTX-A INJECTIONS}

Gage (1991) advised that, whenever possible, surgical intervention should be postponed until the gait is mature. Both from our own research and from the scientific literature, it can be concluded that many research groups are convinced that mature, adult gait is achieved after the age of 6 years in normal children (Pellico et al., 1995). This is at least 4-5 years after acquiring independent gait. Hence, for a large proportion of disabled children, who develop gait at a more advanced age, it is suggested that such patients should receive conservative (i.e. not surgical) treatment until the age of 8-10 years. When this conservative therapy is limited to physiotherapy and the use of orthoses, the dynamic contractures often progress to fixed contractures and even skeletal deformations, causing severe biomechanical lever-arm dysfunction. However, when these therapies are complemented by a treatment for the spasticity, such as BTX-A injections, it is hoped that the consequences of the persistent hypertonicity can be limited, thereby postponing surgery until the appropriate age, or maybe even avoiding surgery altogether for certain patients.

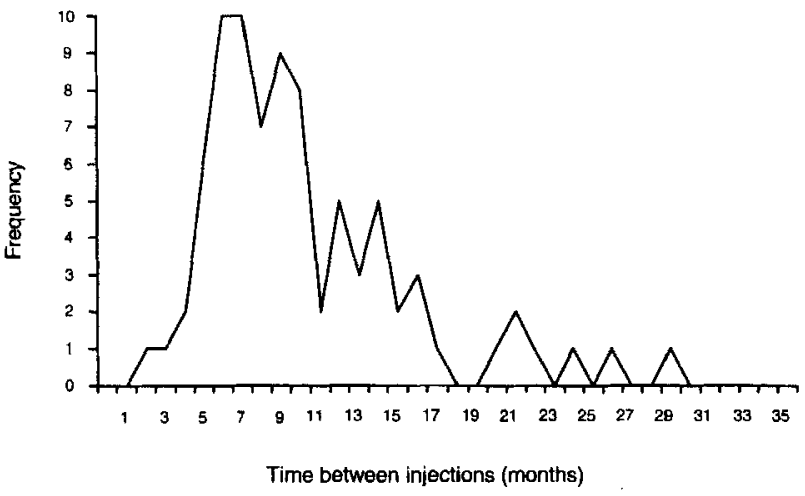

FIGURE 2. Frequency of time between repeat botulinum toxin type A injections $(n=82)$ in 64 children with cerebral palsy $(n=82)$

From functional and clinical post-treatment evaluations, it is clear that the earlier BTX-A treatment is started, the better the outcome is likely to be (Cosgrove, 1994). The general indication for BTX-A injections is 'the presence of a dynamic contracture, interfering with function, in the absence of a fixed myostatic contracture' (Boyd and Graham, 1997).

Between February 1996 and April 1999, we administered BTX-A injections to 316 patients at the University Hospital of Pellenberg. At the time of the first injection, patients had a mean age of 6.5 years [standard deviation (SD), 3.9 years; age range, 1.5-25 years] and a median age of 5.5 years (Figure 1). Treatment included both multilevel and single-level injections; the single-level treatment mainly consisted of BTX-A injections in the iliopsoas, which in older patients was frequently combined with multilevel surgery, thereby explaining the use of BTX-A injections in patients up to the age of 25 years. From Figure 1, it can be seen that the main age group for multilevel BTX-A injections was 2.5-7 years.

Sixty-four CP patients received one or more reinjection sessions ( 50 had two injections, 11 had three injections, two patients received four injections and one patient had five injections). The mean duration between injection sessions for all patients was 11.2 months (SD, 5.3 months), with a median duration of 10 months (Figure 2).

For a sub-group of 156 children given BTX-A treatment between April 1997 and October 1998, more detailed information has been collated and this is presented in the remainder of this article: $58 \%$ ( $n$ $=91$ ) of these injections were given to children with diplegia, $25 \%(n=39)$ to those with hemiplegia and $17 \%(n=26)$ to those with quadriplegia. 


\section{PATIENT SELECTION}

Objective observation of gait is thought to be a decisive factor in the 'fine-tuning' of BTX-A treatment, and, hence, gait analysis plays a crucial role in the identification of target muscles.

All patients treated with BTX-A injections at the University Hospital of Pellenberg are evaluated by full gait analysis, which includes three-dimensional kinematics and kinetics, and surface electromyography (EMG) of seven lower limb muscles. In younger children ( $<4$ years), the gait analysis is limited to normal video recording. Gait analysis is always completed before and after injection, and in combination with an extended clinical examination that includes evaluation of mobility, spasticity, strength and selectivity. An additional evaluation of muscle tone and length is performed under general anaesthesia immediately prior to injection.

\section{MULTILEVEL VERSUS SINGLE-LEVEL TREATMENT}

Based on the results of gait analysis and clinical examination, the necessity for multilevel treatment with BTX-A became apparent. The same principles as those used for multilevel surgery were applied in the selection of target muscles, all treatment being focused on the optimal alignment of the legs and pelvis.

Normal gait has the following characteristics: sufficient stability in stance, adequate foot clearance in swing, appropriate prepositioning in terminal swing, adequate step length and energy conservation (Gage, 1991). Since a pathological gait will lack some of these characteristics, the aim of therapy is to reestablish these basic components of normal gait.

Multilevel BTX-A treatment will aim specifically at improving stability. Using BTX-A treatment, the aim is to establish both a flat foot position in stance and an improved prepositioning of the foot in terminal swing. The latter may, for example, be obtained after a multilevel BTX-A treatment of the hamstrings and gastrocnemius, resulting in improved knee extension at initial contact combined with an improved ankle position in the prepositioning of the foot.

Intensive physiotherapy can work on functionality after a multilevel BTX-A treatment. In cases where a reasonable, selective control of the ankle dorsiflexors was present before BTX-A treatment, the prepositioning of the feet is noticeably enhanced after BTX-A treatment of the calf muscles. Improved prepositioning of the feet and improved knee extension and ankle dorsiflexion at initial contact will usually also be favourable for step length.

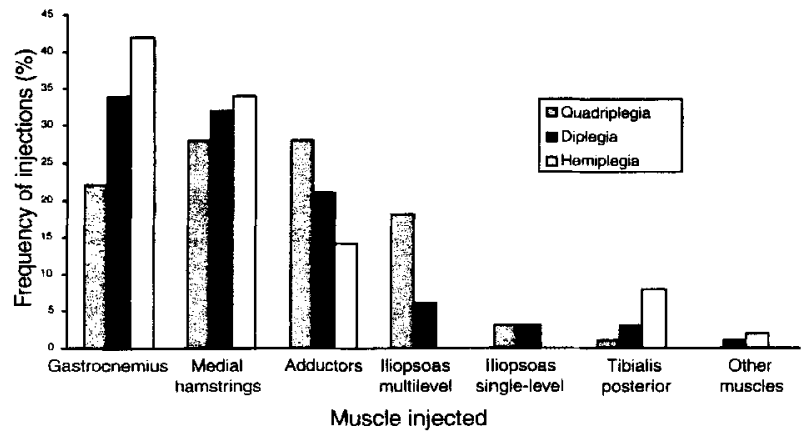

FIGURE 3. Frequency of botulinum toxin type A injections into each muscle, expressed as a percentage of the total number of injections performed between April 1997 and October 1998 in 156 children with cerebral palsy

Figure 3 presents the frequency of injections in each muscle, expressed as a percentage of the total number of injections. Clearly, the gastrocnemius and medial hamstrings are the most frequently injected muscle for children with diplegia and hemiplegia. However, in children with quadriplegia, the medial hamstrings and the adductors are the most frequently injected, followed by the gastrocnemius.

The frequency of the different combinations of single-level and multilevel BTX-A treatments performed between April 1997 and October 1998 as a percentage of the total number of BTX-A treatments is shown in Table 1. For children with hemiplegia, BTX-A treatment most frequently involved injection of the gastrocnemius and medial hamstrings. For children with diplegia and quadriplegia, this combination was often extended to the adductors. For children with quadriplegia, all the muscles in a three-level (gastrocnemius, medial hamstrings, adductors and iliopsoas) or two-level combination excluding the gastrocnemius (i.e. hamstrings, adductors and iliopsoas) were frequently treated.

\section{DOSE OF BTX-A AND INJECTION TECHNIQUES}

\section{Dose of BTX-A}

Using the multilevel approach already described to administer BTX-A, it was necessary to gradually increase the dose of BTX-A to determine the optimal dose per selected muscle. For a child with minor spasticity at the distal muscle groups, an adequate BTX-A treatment would involve injection of the calf muscles only (in our clinic, about $8-9 \%$ of treatments, as shown in Table 1). In contrast, for a child with more severe spasticity, the optimal dose of BTX-A 
TABLE 1. Distribution (percentage) of the combination of target muscles injected with botulinum toxin type A (BTX-A) during one session in 156 children with diplegia, hemiplegia or quadriplegia

\begin{tabular}{lccc}
\hline & & Patients with \\
\cline { 2 - 3 } Muscles injected with BTX-A in one session & diplegia (\%) & hemiplegia (\%) & quadriplegia (\%) \\
\hline Gastrocnemius & 9 & 8 & 2 \\
Gastrocnemius, medial hamstrings & 19 & 38 & 26 \\
Gastrocnemius, medial hamstrings, adductors & 36 & 0 \\
Gastrocnemius, medial hamstrings, adductors, iliopsoas & 5 & 22 \\
Gastrocnemius, medial hamstrings, adductors, tiblalis posterior & 1 & 0 \\
Gastrocnemius, medial hamstrings, tibialis posterior & 4 & 0 \\
Gastrocnemius, tibialis posterior & 0 & 10 & 0 \\
Gastrocnemius, soleus, medial hamstrings, tibialis posterior & 0 & 4 & 0 \\
Gastrocnemius, soleus, medial hamstrings, adductors & 0 & 3 \\
Gastrocnemius, medial hamstrings, tensor fasciae latae & 4 & 3 & 0 \\
Gastrocnemius, medial hamstrings, iliopsoas & 0 & 3 & 0 \\
Gastrocnemius, adductors, iliopsoas & 1 & 0 & 0 \\
Gastrocnemius, adductors & 5 & 0 & 0 \\
Medial hamstrings, adductors & 6 & 0 & 0 \\
Medial hamstrings, adductors, iliopsoas & 7 & 0 \\
lliopsoas (often combined with multilevel surgery) & 1 & 0 \\
lliopsoas, adductors & 1 & 0 & 0 \\
lliopsoas, rectus femoris & & 0 & 0 \\
\hline
\end{tabular}

Values may not add up to $100 \%$ because of the effect of 'rounding'.

should be administered to each of the target muscles involved.

Each $100 \mathrm{U}$ vial of BOTOX ${ }^{\oplus}$ (Allergan Inc., Irvine, CA, USA) was constituted with $2 \mathrm{ml}$ saline. The dose of BTX-A $\left(\right.$ BOTOX $\left.^{\oplus}\right)$ for all patients ranged from 2 to $29 \mathrm{U} / \mathrm{kg}$ body weight (bw), with mean doses of $19.4 \mathrm{U} / \mathrm{kg}$ bw for children with diplegia, $13.0 \mathrm{U} / \mathrm{kg}$ bw for those with hemiplegia and $20.4 \mathrm{U} / \mathrm{kg}$ bw for those with quadriplegia. The frequency of administration of the different doses for all 156 patients is illustrated in Figure 4. BTX-A doses of less than $6 \mathrm{U} / \mathrm{kg} \mathrm{bw}$ were used for isolated injections of the iliopsoas, often in combination with multilevel surgery. As could be expected, the doses for children with hemiplegia were about one-half those used for the diplegic children.

For each selected muscle, BTX-A was injected at multiple sites in order to distribute the amount evenly within the muscle, for a better effect with the product, and to prevent the possibility of the toxin reaching the systemic circulation. The maximal dose per injected site was $50 \mathrm{U} \mathrm{BOTOX}^{\oplus}$, to a maximum total dose of 20-25 U/kg bw. The number of injection sites selected for a CP child were usually: four for the gastrocnemius, four or more for the hamstring, and two or three each for the adductors, tibialis posterior and iliopsoas. No major side effects were reported using this technique.

The following doses of BOTOX ${ }^{\circledast}$ were indicated for use in the specified muscles: $6-8 \mathrm{U} / \mathrm{kg}$ bw for the gastrocnemius, 4-6 U/kg bw for the hamstrings, 2-4 U/kg bw for the adductors, $2-4 \mathrm{U} / \mathrm{kg}$ bw for the iliopsoas, and $2-3 \mathrm{U} / \mathrm{kg}$ bw for the tibialis posterior. These dose guidelines were then fine-tuned on a patient-by-patient basis following objective evaluations of the dynamic functional capacity of each child, using full gait analysis and clinical examinations. In particular, dynamic EMG has a useful role in finetuning the dosage. If a muscle was only mildly involved, then lower BTX-A doses were injected into that muscle. In exceptional cases, the dose was increased, particularly when one muscle appeared to have a major impact on the gait pattern of the child. This is illustrated in Table 2, which contains the percentage distribution of applied doses per muscle for the treatment of children with diplegia, hemiplegia and quadriplegia, respectively. These data highlight the fact that higher doses are injected into the gastrocnemius for children with hemiplegia compared with those with diplegia and quadriplegia. Conversely, children with quadriplegia receive higher BTX-A doses in the hamstrings, adductors and iliopsoas compared with those with diplegia and hemiplegia. None of the 39 children with hemiplegia in this study received injections in the iliopsoas.

\section{Injection technique}

In our clinic, BTX-A is always administered under general anaesthesia, particularly when used as a multi- 


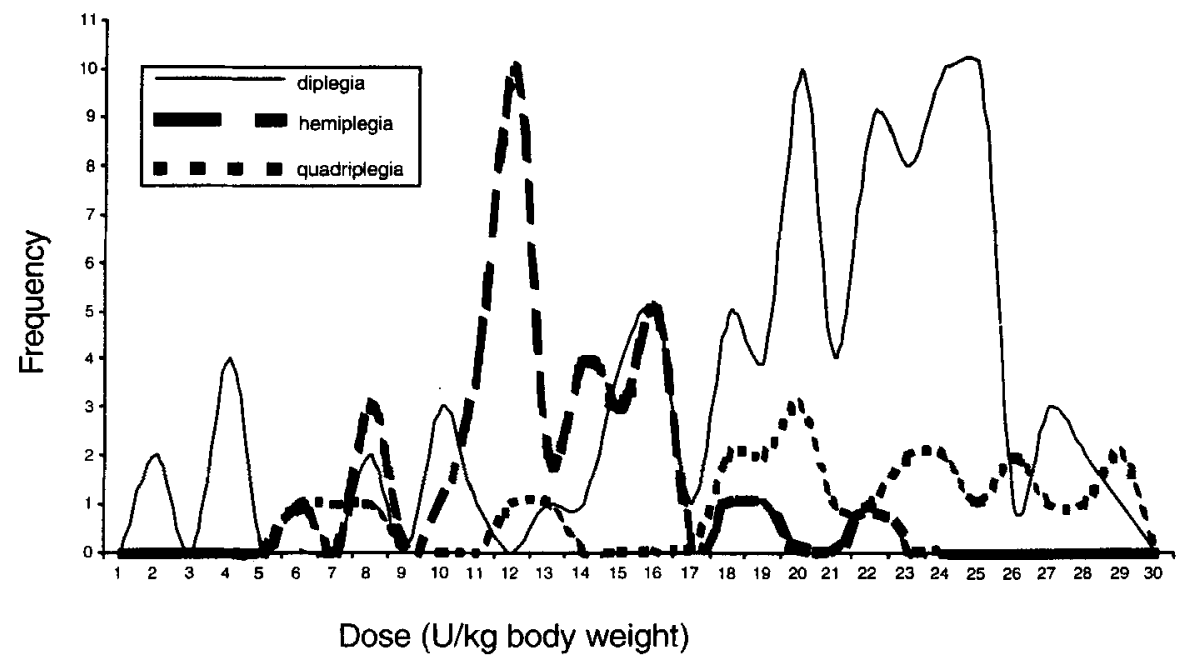

FIGURE 4. Frequency of administration of the different doses of botulinum toxin type $A$ (BOTOX ${ }^{\circledR}$ ) for 156 children with diplegia, hemiplegia and quadriplegia

TABLE 2. The percentage distribution of BOTOX doses administered in each muscle type in 156 cerebral palsy (CP) children with diplegia, hemiplegia and quadriplegia, treated between April 1997 and October 1998

\begin{tabular}{|c|c|c|c|c|c|c|c|c|c|c|}
\hline \multirow[b]{2}{*}{ Muscle } & \multirow[b]{2}{*}{ CP child } & \multicolumn{9}{|c|}{ Dose (U/kg) } \\
\hline & & 1 & 1.5 & 2 & 3 & 4 & 5 & 6 & 7 & 8 \\
\hline \multirow[t]{3}{*}{ Gastrocnemius } & Diplegia (\%) & 0 & 0 & 0 & 0 & 13 & 12 & 42 & 17 & 16 \\
\hline & Hemiplegia (\%) & 0 & 0 & 0 & 0 & 8 & 3 & 25 & 28 & 36 \\
\hline & Quadriplegia (\%) & 0 & 0 & 14 & 7 & 7 & 21 & 34 & 10 & 7 \\
\hline \multirow[t]{3}{*}{ Hamstrings } & Diplegia (\%) & 0 & 0 & 7 & 7 & 54 & 17 & 8 & 4 & 3 \\
\hline & Hemiplegia (\%) & 3 & 0 & 7 & 0 & 57 & 17 & 13 & 0 & 3 \\
\hline & Quadriplegia (\%) & 0 & 0 & 11 & 0 & 35 & 49 & 5 & 0 & 0 \\
\hline \multirow[t]{3}{*}{ Adductors } & Diplegia (\%) & 16 & 9 & 38 & 25 & 9 & 1 & 0 & 0 & 2 \\
\hline & Hemiplegia (\%) & 0 & 0 & 75 & 9 & 8 & 0 & 0 & 0 & 0 \\
\hline & Quadriplegia (\%) & 5 & 0 & 20 & 51 & 24 & 0 & 0 & 0 & 0 \\
\hline Iliopsoas & Diplegia (\%) & 12 & 0 & 79 & 9 & 0 & 0 & 0 & 0 & 0 \\
\hline \multirow[t]{2}{*}{ (multilevel) } & Hemiplegia (\%) & 0 & 0 & 0 & 0 & 0 & 0 & 0 & 0 & 0 \\
\hline & Quadriplegia (\%) & 17 & 0 & 46 & 37 & 0 & 0 & 0 & 0 & 0 \\
\hline lliopsoas & Diplegia (\%) & 0 & 0 & 61 & 8 & 31 & 0 & 0 & 0 & 0 \\
\hline \multirow[t]{2}{*}{ (single-level) } & Hemiplegia (\%) & 0 & 0 & 0 & 0 & 0 & 0 & 0 & 0 & 0 \\
\hline & Quadriplegia (\%) & 0 & 0 & 0 & 50 & 50 & 0 & 0 & 0 & 0 \\
\hline \multirow[t]{3}{*}{ Tibialis posterior } & Diplegia (\%) & 0 & 0 & 54 & 20 & 26 & 0 & 0 & 0 & 0 \\
\hline & Hemiplegia (\%) & 0 & 0 & 85 & 15 & 0 & 0 & 0 & 0 & 0 \\
\hline & Quadriplegia (\%) & 0 & 0 & 100 & 0 & 0 & 0 & 0 & 0 & 0 \\
\hline
\end{tabular}

Values may not add up to $100 \%$ because of the effect of 'rounding'.

level treatment, as this also enables a supplementary clinical evaluation under anaesthesia to be performed.

Correct needle placement (usually 26 gauge $x$ $23 \mathrm{~mm}$ and 22 gauge $\times 30 \mathrm{~mm}$ for injection of the iliopsoas in an older child) was checked by moving the articulation above or beyond the muscle while the muscle was stretched and observing the needle motion. 


\section{FOLLOW-UP TREATMENT AFTER BTX-A INJECTIONS}

The duration of effect of BTX-A treatment was mainly determined by the follow-up treatment consisting of, for example, serial casting, day and night splinting, and physiotherapy.

There is an on-going discussion as to the optimal combination for BTX-A injections and serial casting for CP children. Since BTX-A is effective on spasticity and casting works on early contractures, the combination of BTX-A injections and casting usually results in better tolerance of casting and easier correction of muscle contractures. However, as any neuronal activity improves uptake of the toxin, immobilisation after BTX-A injections may not be the ideal solution. A study is therefore ongoing at the University Hospital of Pellenberg to define the optimal strategy for combining BTX-A treatment and serial casting in spastic $\mathrm{CP}$ children with mild contractures. Over the past 2 years, $49 \%$ of BTX-A treatments were immediately followed by $2-4$ weeks of serial casting. Hence, the ongoing prospective study was set up to define the optimal combination and timing of BTX-A injections and serial casting (both before and/or after BTX-A treatment).

After BTX-A injections, the use of night splinting and day orthoses appears to be a critical factor in influencing the long-term effects of BTX-A treatment: both their role in preserving muscle length and their provision of stability to distal joints are thought to be important. This enables selective training of more proximal muscle groups ('Target Training'). Of the 156 children studied, $44 \%$ were fitted with day orthoses [ankle-foot orthosis (AFO)] before BTX-A treatment and $83 \%$ were fitted with day orthoses posttreatment. Night splinting increased from $30 \%$ before treatment to $76 \%$ after treatment.

As previously mentioned, BTX-A treatment needs a multidisciplinary approach. In this process, the role of the physiotherapist is crucial to guiding the child towards motor learning and exploiting newly retrieved motor function. In our clinic, $30 \%$ of treated patients had physiotherapy once or twice per week, $39 \%$ had physiotherapy three or four times per week and $28 \%$ received physiotherapy five to seven times per week. During the period of 3-4 months of suppressed hypertonia post-injection, a temporary increase in physiotherapy is advised in order to profit maximally from the benefits of BTX-A injections.

\section{FUNCTIONAL OUTCOME}

A retrospective study of multilevel BTX-A treatment in combination with short leg casting and orthotic management of the gait was performed on a cohort of 33 children with CP. For inclusion in the study, patients had to be ambulant (without walking aids) with a diagnosis of spastic $\mathrm{CP}$, they had to complete a full barefoot gait analysis (using a six-camera Vicon data-capturing system and two Advanced Mechanical Technology, Inc., force plates for collection of kinematic and kinetic data, and using surface EMG for seven lower extremity muscles on both limbs), and have a clinical examination before and 2 months after BTX-A treatment.

Twelve children (seven with diplegia and five with hemiplegia) had multilevel BTX-A treatment combined with proper orthotic management (see later); 15 patients (six with diplegia and nine with hemiplegia) had multilevel BTX-A treatment combined with proper orthotic management and short leg casting immediately after BTX-A injections; and six patients (three with diplegia and three with hemiplegia) had multilevel BTX-A treatment combined with short leg casting before BTX-A injections and proper orthotic management after BTX-A treatment. Orthotic management after BTX-A injections involved day orthoses (AFO) and night splinting. All patients had physiotherapy sessions before and after BTX-A injections. Gait data were processed using the Vicon Clinical Manager software (Oxford Metrics, Oxford, UK). Data analysis included comparisons of different gait parameters using the Student $t$-test (paired, two-sided). Variables for comparison were selected from the kinematic (joint angles), kinetic (internal moments and power) and EMG (timing of activity) results.

A multilevel BTX-A treatment combined with orthotic management resulted in a significantly more stable pelvis during stance (especially in the frontal and transversal plane) $(P<0.05)$, with the most marked improvements being observed in patients who did not have short leg casting or who had casting before treatment. Children who had BTX-A injections without casting showed a significantly higher hip extension at terminal stance compared with their hip kinematics before treatment $(P<0.05)$. The incidence of internal hip rotation in stance and swing (frequently seen before treatment), decreased significantly $(P<0.01)$ for all patients after treatment. All children, except for those who had serial casting before BTX-A injections, showed significantly improved knee kinematics in stance after treatment (decrease of knee hyper extension or decrease of crouch gait $)(P<0.05)$. The frequency of a pathological plantar flexion/knee extension couple decreased significantly after BTX-A treatment in all patients.

Power generation at the ankle joint at push-off increased after BTX-A injections in all patients. 
The frequency of adequate tibialis anterior activity during terminal swing and loading response increased after BTX-A treatment compared with the EMG results from the pre-treatment gait analysis. Premature gastrocnemius activity in stance is still common after BTX-A injections, although most patients demonstrated a lower level of gastrocnemius activity at the initial follow-up post-treatment.

In summary, a complete multilevel BTX-A treatment, combined with orthotic management and serial casting if necessary, is very useful in the management of spasticity in children with CP. Some differences in the resulting gait patterns were observed between children who did not have serial casting combined with BTX-A treatment, and those who had casting either before or after BTX-A treatment.

\section{CONCLUSIONS}

The present integrated approach to BTX-A treatment originates from the idea that in order to improve functional outcome, treatment had to involve all levels simultaneously. As a consequence of this, the administered dose of BTX-A was increased to achieve an optimal dose per injected muscle.
Our results indicate that this multidisciplinary approach is successful in correcting major gait deviations in children with $\mathrm{CP}$, and hence in preventing major lever-arm dysfunctions.

Regardless of the dose administered in our clinic, no major side effects of BTX-A were noted. An increase in the number of BTX-A injection sites per muscle appears to be crucial in compensating for the higher doses. This current approach appears to prolong the duration of the BTX-A treatment, resulting in an inter-injection interval of approximately 1 year.

\section{REFERENCES}

Boyd R, Graham HK (1997). Botulinum toxin A in the management of children with cerebral palsy: indication and outcome. Eur J Neurol 4 (suppl 2):S15-S22.

Cosgrove AP (1994). Botulinum toxin in the management of the lower limb in cerebral palsy. Dev Med Child Neurol 36:386-396.

Gage JR (1991). Gait Analysis in Cerebral Palsy. London: MacKeith Press.

Pellico LG, Torres RR, Mora CD (1995). Changes in walking pattern between five and six years of age. Dev Med Child Neurol 37:800-806. 